\title{
CAMBIOS EN LOS VALORES PROFESIONALES ENFERMEROS DURANTE LA PANDEMIA POR COVID-19
}

\section{Changes in Nursing Professional Values during the COVID-19 Pandemic}

\author{
María GONZÁLEZ-GARCÍA ${ }^{\mathrm{a}, \mathrm{b}} ;$ Ana FERNÁNDEZ-FEITOº, Alberto LANA $^{\mathrm{b}, \mathrm{c}}$ \\ ${ }^{a}$ Servicio de Salud del Principado de Asturias. Hospital Universitario Central de Asturias. 'Instituto de \\ Investigación Sanitaria del Principado de Asturias (ISPA). 'Departamento de Medicina. Facultad de Medicina y \\ Ciencias de la Salud. Universidad de Oviedo (España).
}

Correo electrónico: mariagonzalezgarcia13@gmail.com

Fecha de recepción: 3 de septiembre de 2020

Fecha de aceptación: el 5 de septiembre de 2020

Fecha de publicación: 29 de enero de 2021

\begin{abstract}
Resumen
La crisis sanitaria provocada por la pandemia por la COVID-19 ha puesto en juego los valores profesionales. El objetivo de este estudio fue describir los cambios en los valores profesionales de enfermeros/as de atención especializada durante los primeros meses de la pandemia en Asturias (España). Se seleccionó una muestra de 55 profesionales de enfermería de cuidados críticos, urgencias, plantas de hospitalización y gestión. Se administró el cuestionario validado de Escala de Valores Profesionales de Enfermería (26 ítems), que consta de 3 dimensiones: ética (9 ítems), compromiso (8 ítems) y dominio profesional (9 ítems). Para cada valor, se solicitó que indicaran si hubo o no cambios (negativos o positivos). En general, se detectaron cambios positivos en los valores profesionales de compromiso y de dominio profesional durante la primera ola de la pandemia. Los valores que acumularon más cambios positivos fueron "buscar formación para actualización", "proteger la salud y seguridad de la población" y "aceptar responsabilidad de la práctica". Los valores éticos de los enfermeros/as permanecieron más estables.

Palabras clave: infecciones por coronavirus; enfermería; ética en enfermería; valores profesionales.
\end{abstract}

\section{Abstract}

The health crisis caused by the COVID-19 pandemic has challenged the professional values. The aim of the study was to describe the changes in the professional values of hospital nurses 
during the first months of the pandemic in Asturias (Spain). A sample of 55 critical care, emergency, hospitalization units and management nurses were selected. The validated Professional Nursing Values Scale (26 items) was used, which consists of 3 dimensions: ethics ( 9 items), professional commitment ( 8 items) and professional mastery ( 9 items). For each value, it was asked to indicate whether there were changes (positive or negative) or not. Overall, positive changes in values of professional commitment and mastery were detected during the first wave of the pandemic. The values that showed the most positive changes were "Find additional training to update one's knowledge and skills", "Protect the health and safety of the population" and "Accept responsibility for one's practice". The nurses ethical values remained more steady.

Keywords: coronavirus infections; nursing; nursing ethics; professional values.

\section{INTRODUCCIÓN}

La pandemia mundial provocada por la enfermedad por SARS-CoV-2 (COVID-19) está desafiando los sistemas sanitarios de todo el mundo. Durante los primeros meses de su evolución, los trabajadores de la salud fueron duramente golpeados por la crisis sanitaria. Al desconocimiento inicial de la enfermedad y el elevado grado de incertidumbre clínica, se añadió tener que adaptarse a entornos de trabajo totalmente nuevos, en los que hubo escasez de recursos humanos y materiales, abrumadora presión social y elevado riesgo de contagio ${ }^{1}$. A pesar de soportar un alto nivel de estrés laboral y personal ${ }^{2}$, los trabajadores de salud mostraron gran dedicación profesional y aceptaron la necesidad de ponerse en riesgo y trabajar hasta la extenuación en entornos clínicos muy complejos ${ }^{3}$. De todos los trabajadores de la salud, el colectivo enfermero es el más numeroso, y ha sido clave por su labor en todos los niveles asistenciales ${ }^{4}$.

En crisis de gravedad extrema, los valores profesionales permiten a los trabajadores continuar, pero estos pueden resquebrajarse si los golpes repetidos exceden su solidez $z^{5}$. Los valores profesionales de enfermería hacen referencia al conjunto de valores inherentes a la práctica de esta profesión ${ }^{6}$, donde se incluyen valores éticos, valores relacionados con la ejecución y destreza de los cuidados de enfermería y con el compromiso con la competencia profesional y el avance de la profesión ${ }^{7}$. Se sabe que algunos factores pueden modificar la percepción sobre estos valores, como la experiencia profesional y/o el entorno de trabajo ${ }^{8}$. Por lo tanto, es razonable pensar que enfrentarse a una crisis sanitaria de esta magnitud ha podido afectar a los valores profesionales enfermeros.

El objetivo fue describir los cambios en los valores profesionales de enfermeros/as de atención especializada durante los primeros meses de la pandemia por COVID-19 en Asturias (España).

\section{MATERIAL y MÉTOdos}

\section{Diseño y participantes}

Estudio descriptivo transversal de un grupo de enfermeros/as del Servicio de Salud del Principado de Asturias (SESPA).

En junio de 2020 se seleccionaron de forma oportunista a un grupo de 55 enfermeros/as que trabajaban en cuidados críticos, urgencias, plantas de hospitalización y gestión. El principal centro de trabajo de esta muestra fue el Hospital Universitario Central de Asturias (HUCA), que es el hospital de referencia de Asturias. El HUCA es un hospital público multiespecialidad de tercer nivel, con 1.039 camas y en el que trabajan habitualmente 1.547 enfermeros/as. Para el reclutamiento, primero se seleccionó a un trabajador de cada unidad y se le pidió que invitara a participar a algún otro de su confianza hasta completar la muestra de conveniencia. En el subgrupo de profesionales en puestos de gestión se incluyeron puestos de supervisión de las 
unidades clínicas y cargos de gestión tanto del HUCA como de otros hospitales de la región en Asturias. Todos los participantes otorgaron consentimiento informado. El estudio contó con la aprobación del Comité de Ética para la Investigación del Principado de Asturias (Referencia: Proyecto 2020.381)

\section{Instrumento y variables del estudio}

Los participantes respondieron a un cuestionario online que constó de dos partes. La primera de ellas recogió variables sociodemográficas (sexo y edad) y laborales. Concretamente, se obtuvo información de la unidad en la que trabajó durante el periodo marzo-junio 2020, la experiencia profesional (años), la frecuencia de agotamiento emocional (a diario, frecuentemente, ocasionalmente o nunca) y el nivel de satisfacción laboral medido según una escala numérica de 1 (muy insatisfecho) a 10 (muy satisfecho). En la segunda parte, se administró el cuestionario de Escala de Valores Profesionales de Enfermería (EVPE) adaptado y validado en España ${ }^{9,10}$. Este cuestionario consta de 26 ítems agrupados en 3 factores: ética (9 ítems), compromiso profesional (8 ítems) y dominio profesional (9 ítems). La consistencia interna de cada factor es adecuada (alpha de Cronbach: 0,77, 0,71 y 0,70 , respectivamente). Se solicitó a los participantes que valoraran en qué medida la pandemia por COVID-19 había producido cambios en estos valores según las siguientes opciones: bastante o muy negativos, un poco negativos, sin cambios, un poco positivos, bastante o muy positivos.

\section{Análisis de datos}

Se realizó un análisis descriptivo utilizando frecuencias absolutas y relativas (\%). Para una mayor sencillez en la interpretación de los resultados, las cinco categorías de la EVPE se transformaron en tres fusionando las categorías extremas, de forma que sólo se consideraron "cambios negativos", "sin cambios" o "cambios positivos". Los análisis fueron realizados utilizando el programa estadístico SPSS (IBM Corp.; Nueva York).

\section{RESULTADOS}

Respondieron a la encuesta online 55 profesionales de enfermería. Las características de los participantes se muestran en la tabla 1 . Casi 9 de cada 10 fueron mujeres, con una edad media de 39,9 años (DE: 9,41) y experiencia profesional media de 16,7 años (DE: 9,27). La mayor parte manifestó estar frecuentemente $(43,6 \%)$ u ocasionalmente $(40,0 \%)$ agotado desde el punto de vista emocional.

Tabla 1. Características de los participantes

\begin{tabular}{|l|c|}
\hline & $\mathbf{n}(\%)$ \\
\hline Sexo & $7(12,7)$ \\
\hline Hombre & $48(87,3)$ \\
\hline Mujer & \\
\hline Edad & $28(50,1)$ \\
\hline$<40$ años & $27(49,9)$ \\
\hline$\geq 40$ años & \\
\hline Experiencia profesional & $32(58,2)$ \\
\hline$<20$ años & $23(41,8)$ \\
\hline$\geq 20$ años & \\
\hline Unidad de trabajo & $20(36,4)$ \\
\hline Cuidados críticos & $14(25,5)$ \\
\hline Puestos de gestión & $12(21,8)$ \\
\hline Unidad de hospitalización & $9(16,4)$ \\
\hline Urgencias & \\
\hline Agotado emocionalmente & $24(14,5)$ \\
\hline A diario & \\
\hline Frecuentemente & \\
\hline Ocasionalmente & \\
\hline Nunca & \\
\hline
\end{tabular}


Los participantes reconocieron cambios en muchos de los valores profesionales considerados (tabla 2). En conjunto, los valores que más cambiaron fueron los de compromiso, seguidos de los de dominio. En todos los valores hubo una mayor frecuencia de cambios positivos que negativos, excepto en "Involucrarse en autoevaluación" y "Promover profesionalidad con estudiantes". Los valores que acumularon más cambios positivos fueron, en orden decreciente: "Buscar formación para actualización" (68,5\%), "Proteger la salud y seguridad de la población" (67,3\%) y "Aceptar responsabilidad de la práctica" (61,8\%).

Tabla 2. Cambios en los valores profesionales

\begin{tabular}{|c|c|c|c|}
\hline & $\begin{array}{c}\text { Cambios } \\
\text { negativos } \\
\text { n (\%) }\end{array}$ & $\begin{array}{c}\text { Sin } \\
\text { cambios } \\
\text { n (\%) }\end{array}$ & $\begin{array}{c}\text { Cambios } \\
\text { positivos } \\
\text { n (\%) }\end{array}$ \\
\hline \multicolumn{4}{|l|}{ Valores éticos } \\
\hline $\begin{array}{l}\text { Asumir la responsabilidad de satisfacer las necesidades de salud } \\
\text { de una población culturalmente diversa }\end{array}$ & $2(3,6)$ & $24(43,6)$ & $29(52,7)$ \\
\hline Proteger los derechos morales y legales de los pacientes & $8(14,5)$ & $20(36,4)$ & $27(49,1)$ \\
\hline Actuar como defensora de los derechos del paciente & $5(9,1)$ & $30(54,5)$ & $20(36,4)$ \\
\hline Proveer atención sin prejuicios a pacientes con estilos de vida diversos & $2(3,6)$ & $36(65,5)$ & $17(30,9)$ \\
\hline Salvaguardar el derecho de los pacientes a la intimidad & $5(9,1)$ & $26(47,3)$ & $24(43,6)$ \\
\hline $\begin{array}{l}\text { Hacer frente a los profesionales con prácticas inapropiadas o } \\
\text { cuestionables }\end{array}$ & $6(10,9)$ & $25(45,5)$ & $24(43,6)$ \\
\hline $\begin{array}{l}\text { Proteger los derechos de los pacientes participantes en procesos de } \\
\text { investigación }\end{array}$ & $2(3,7)$ & $39(72,2)$ & $13(24,1)$ \\
\hline $\begin{array}{l}\text { Ejercer la profesión teniendo en cuenta principios de fidelidad y } \\
\text { respeto a la persona }\end{array}$ & $2(3,6)$ & $30(54,5)$ & $23(41,8)$ \\
\hline Garantizar la confidencialidad del paciente & $5(9,1)$ & $29(52,7)$ & $21(38,2)$ \\
\hline \multicolumn{4}{|l|}{ Valores de compromiso profesional } \\
\hline Proteger la salud y la seguridad de la población & $8(14,5)$ & $10(18,2)$ & $37(67,3)$ \\
\hline Participar en decisiones sobre la distribución de recursos & $14(25,5)$ & $10(18,2)$ & $31(56,4)$ \\
\hline Participar en procesos de revisión entre colegas & $9(16,4)$ & $18(32,7)$ & $28(50,9)$ \\
\hline Iniciar acciones para la mejora de los entornos del ejercicio profesional & $11(20,0)$ & $17(30,9)$ & $27(49,1)$ \\
\hline $\begin{array}{l}\text { Hacer avanzar la profesión a través de involucrarse activamente en } \\
\text { actividades relacionadas con la salud }\end{array}$ & $11(20,0)$ & $17(30,9)$ & $27(49,1)$ \\
\hline $\begin{array}{l}\text { Reconocer el papel de las organizaciones profesionales en la } \\
\text { construcción de políticas de salud }\end{array}$ & $16(29,1)$ & $14(25,5)$ & $25(45,5)$ \\
\hline $\begin{array}{l}\text { Participar en investigación en enfermería y llevar a la práctica los } \\
\text { hallazgos }\end{array}$ & $13(23,6)$ & $25(45,5)$ & $17(30,9)$ \\
\hline Participar en actividades de asociaciones profesionales & $10(18,2)$ & $38(68,1)$ & $7(12,7)$ \\
\hline
\end{tabular}




\begin{tabular}{|c|c|c|c|}
\hline & $\begin{array}{c}\text { Cambios } \\
\text { negativos } \\
\text { n (\%) }\end{array}$ & $\begin{array}{c}\text { Sin } \\
\text { cambios } \\
\text { n (\%) }\end{array}$ & $\begin{array}{c}\text { Cambios } \\
\text { positivos } \\
\text { n (\%) }\end{array}$ \\
\hline \multicolumn{4}{|l|}{ Valores de dominio profesional } \\
\hline Involucrarse en un proceso de autoevaluación continua & $21(38,2)$ & $19(34,5)$ & $15(27,3)$ \\
\hline $\begin{array}{l}\text { Solicitar consulta/colaboración cuando no puede satisfacer las } \\
\text { necesidades del paciente }\end{array}$ & $9(16,4)$ & $25(45,5)$ & $21(38,2)$ \\
\hline Establecer criterios de calidad como guía para la práctica & $11(20,0)$ & $24(43,6)$ & $20(36,4)$ \\
\hline $\begin{array}{l}\text { Promover y mantener niveles de profesionalidad en las actividades } \\
\text { planificadas para estudiantes. }\end{array}$ & $19(34,5)$ & $24(43,6)$ & $12(21,8)$ \\
\hline $\begin{array}{l}\text { Buscar formación complementaria para actualizar sus conocimientos } \\
\text { y habilidades }\end{array}$ & $4(7,4)$ & $13(24,1)$ & $37(68,5)$ \\
\hline $\begin{array}{l}\text { Promover el acceso equitativo a la Atención enfermera y de } \\
\text { salud en general. }\end{array}$ & $8(14,5)$ & $30(54,5)$ & $17(30,9)$ \\
\hline Aceptar la responsabilidad de la propia práctica y responder de ella & $6(10,9)$ & $15(27,3)$ & $34(61,8)$ \\
\hline Mantener la competencia profesional en el ejercicio profesional & $6(10,9)$ & $18(32,7)$ & $31(56,4)$ \\
\hline $\begin{array}{l}\text { Rehusar participar en prácticas que estén en contraposición ética a } \\
\text { los valores profesionales adquiridos }\end{array}$ & $6(10,9)$ & $34(61,8)$ & $15(27,3)$ \\
\hline
\end{tabular}

\section{DISCUSIÓN}

Según los resultados de este estudio, la mayoría de los cambios en los valores profesionales enfermeros fueron positivos e hicieron referencia a valores de compromiso y dominio profesional. Por el contrario, los valores éticos registraron pocos cambios.

Tanto los cambios positivos en unos valores, como la ausencia de cambios en otros, son coherentes con la situación sanitaria y tiene una lectura alentadora. En primer lugar, los cambios positivos en el compromiso y dominio profesional son razonables, ya que se consideran valores flexibles, que en muchos casos dependen del entorno institucional de trabajo ${ }^{8}$. La primera ola pandémica por la COVID-19 expuso profesionalmente unos centros sanitarios usualmente caracterizados por un elevado nivel de burocratización, protocolización y tecnologización, y puso en primera línea a las personas y a la baja tecnología. En concreto, la inédita situación provocó cambios positivos en la actualización de conocimientos y redobló el afán por proteger la salud y garantizar la seguridad de la población, valores en los que se detectaron mayores porcentajes de cambios positivos. Además, hubo un reconocimiento social unánime a los trabajadores de la salud, lo que pudo fortalecer su papel y la sensación de responsabilidad. Los participantes también identificaron un efecto favorable de la pandemia en las decisiones sobre recursos, que es coherente con el papel protagonista de la tecnología de bajo nivel (p.ej. mascarillas, guantes o batas). Precisamente, la disponibilidad de material, aparataje y equipos de protección individual ha sido reconocido como un elemento clave ${ }^{11}, y$ los enfermeros/as tuvieron un importante papel en su gestión ${ }^{12}$.

En segundo lugar, una menor frecuencia de cambios - positivos o negativos- en los valores éticos también era esperable, incluso deseable. Este tipo de valores son necesariamente 
resistentes al cambio, ya que forman el sustento sobre el que arraiga una actividad enfermera netamente humana. A pesar de que la crisis sanitaria llevó a los trabajadores al límite del colapso mental, emocional y laboral, muchos de los valores éticos fundamentales, como defender los derechos de los pacientes, garantizar la confidencialidad o ejercer con fidelidad y respeto, no se vieron alterados. Esto reafirma la importancia que le otorgan los profesionales a la práctica ética durante la prestación de cuidados de enfermería $^{8,13}$. No obstante, que los valores éticos se hayan visto poco alterados no implica que no se hayan producido dilemas éticos de enorme trascendencia durante los primeros meses de pandemia. De hecho, profesionales de la salud de todo el mundo han tenido que enfrentar decisiones con un marcado componente ético, sobre todo en unidades de cuidados intensivos o en la atención a pacientes y familiares al final de la vida ${ }^{14,15}$. Lo positivo es que, al enfrentarse a esos dilemas, parece que lo han hecho utilizando unos valores éticos robustos. En todo caso, sería deseable revisar la atención sanitaria proporcionada en durante la primera ola por COVID-19 con el objetivo de diseñar un marco ético global que facilite la atención de enfermería ante estas situaciones sanitarias excepcionales, tal y como ya han realizado algunas organizaciones profesionales ${ }^{16}$, y que tenga en cuenta el desgaste emocional que supone trabajar en oposición a los valores profesionales ${ }^{5,17}$. Además, dados el aparente afrontamiento eficaz de los enfermeros/as y su experiencia, resulta ineludible su integración en los equipos multidisciplinares encargados de gestionar esta o futuras pandemias ${ }^{5,18}$.

Nuestro estudio fue pionero y preliminar. Valorar como impactan las situaciones sanitarias de gravedad extrema en la ejecución de los valores profesionales de enfermería sigue siendo necesario, en muestras más grandes y haciendo seguimientos a medio y largo plazo. Este conocimiento ayudará a afianzar el marco de referencia sobre el que se basa la actividad profesional diaria de los enfermeros/as, incluyendo la relación entre colegas. Es importante considerar que llegarán nuevas pandemias, y que siempre se llegará tarde para afrontar la primera ola epidémica, por lo que la necesidad de entender su efecto sobre los valores profesionales puede ser recurrente.

Este estudio presenta algunas limitaciones. En primer lugar, no podemos descartar un sesgo en la información derivado de la muestra oportunista, ya que pudieron participar los profesionales con más gana de aportar su experiencia ante la situación vivida. Por tanto, los resultados no pueden extrapolarse a toda la población de enfermeros/as de Asturias. En cualquier caso, pensamos que, de haberse producido este sesgo, lo más probable es que hubieran contestado los profesionales más enfadados con la gestión de la crisis, lo que habría llevado a una visión más negativa de los cambios. En segundo lugar, los resultados de estudios realizados durante el pico de máxima incidencia pudieron verse influidos por la intensa vivencia emocional, tanto desde el punto de vista personal como profesional. Para intentar atenuar este efecto, la recogida de datos se realizó en junio de 2020, cuando se había logrado el control de la primera ola epidémica. Finalmente, la percepción positiva apuntada por una pequeña muestra de profesionales de Asturias no puede ser extrapolada a otras comunidades autónomas con mayor afectación o diferente gestión de la crisis ${ }^{19,20}$.

En conclusión, durante la primera ola de la pandemia por COVID-19 se detectaron cambios en los valores profesionales de los enfermeros/ as de Asturias. La mayoría de las modificaciones fueron positivas y afectaron sobre todo al compromiso profesional. Los valores éticos han permanecido relativamente estables durante la pandemia. Sería recomendable monitorizar la evolución de estos valores profesionales 
CAMBIOS EN LOS VALORES PROFESIONALES ENFERMEROS DURANTE LA PANDEMIA POR COVID-19 MARÍA GONZÁLEZ-GARCÍA; ANA FERNÁNDEZ-FEITO; ALBERTO LANA

enfermeros para conocer los efectos de esta pandemia a medio/largo plazo.

\section{REFERENCIAS}

1. Nagesh S, Chakraborty S. Saving the Frontline Health Workforce Amidst the COVID-19 Crisis: Challenges and Recommendations. J Glob Health. 2020;10(1): 010345.

2. Preti E, Di Mattei V, Perego G, Ferrari F, Mazzetti M, Taranto P, Di Pierro R, Madeddu F, Calati R. The Psychological Impact of Epidemic and Pandemic Outbreaks on Healthcare Workers: Rapid Review of the Evidence. Curr Psychiatry Rep. 2020; 22(8):43.

3. Liu Q, Luo D, Haase JE, Guo Q, Wang XQ, Liu S, Xia L, Liu Z, Yang J, Yang BX. The experiences of health-care providers during the COVID-19 crisis in China: a qualitative study. Lancet. 2020; 8(6):E790-8.

4. Buheji $M$, Buhaid N. Nursing human factor during COVID-19 pandemic. Int J of Nursing Science. 2020;10:12-24.

5.Turale S, Meechamnan $\mathrm{CH}$, Kunaviktikul W. ChaIlenging Times: Ethics, Nursing and the COVID-19 Pandemic. Int Nurs Rev. 2020;67(2):164-7.

6. Rassin M. Nurses' professional and personal values. Nurs Ethics. 2008; 15(5): 614-30.

7. Elliott $A$. Identifying professional values in nusing: an integrative review. Teaching and Learning in Nursing. 2017; 12(3): 201-6.

8. Fernández-Feito A, Palmeiro-Longo MD, Hoyuelos SB, García-Díaz V. How work setting and job experience affect professional nurses' values. Nurs Ethics. 2019; 26(1): 134-47.

9. Basurto SH,Lobato CF, Weis D, De Lorenzo Uriena E, Elsdend CA, Schankc MJ. Nursing professional values: validation of a scale in a Spanish context. Nurse Educ Today 2010; 30(2): 107-12.

10. Basurto Hoyuelos S. Los valores en la profesión enfermera: validación de un cuestionario [tesis doctoral]. Vitoria-Gasteiz: Universidad del País Vasco;2010.
11. Iserson KV. Healthcare Ethics During a Pandemic. West J Emerg Med. 2020;21(3):477-83.

12. Bellver Capella V. Problemas bioéticos en la prestación de los cuidados enfermeros durante la pandemia del COVID-19. Index de Enfermería; 29(1-2): e12909

13. Poorchangizi B, Farokhzadian J, Abbaszadeh A, Mirzaee M, Borhani F. The importance of professional values from clinical nurses' perspective in hospitals of a medical university in Iran. BMC Med Ethics. 2017;18: 20.

14. Robert R, Kentish-Barnes N, Boyer A, Laurent A, Azoulay E, Reignier J. Ethical dilemmas due to the Covid-19 pandemic. Ann. Intensive Care. 2020; 10:84.

15. Morley G, Grady C, McCarthy J, Ulrich CM. Covid19: Ethical Challenges for Nurses. Hastings Cent Rep. 2020;50: 35-9.

16. Comisión de ética y deontología del consejo de enfermería de la Comunitat Valenciana. Aspectos éticos de la enfermería en la crisis de pandemia por Covid-19 [Monografía en internet]. Valencia: Consejo de enfermería de la Comunitat Valenciana; 2020[acceso 30 de Julio de 2020].

17. Martín Ferreres ML. La presencia de los valores en la práctica enfermera [tesis doctoral]. Barcelona: Universitat Internacional de Catalunya; 2017.

18. Bambi S, Lozzo P, Lucchini A. New Issues in Nursing Management During the COVID-19 Pandemic in Italy. Am J Crit Care. 2020; 29(4): e92-3.

19. COVID-19 [sede web]. Madrid: Gobierno de España; 2020 [acceso 30 de Julio de 2020]. Evolución pandemia.

20. Centro de Coordinación de Alertas y Emergencias Sanitarias. Actualización no 125. Enfermedad por el coronavirus (COVID-19). 03.06.2020 [Monografía en internet]. Madrid: Gobierno de España; 2020 [acceso 30 de Julio 2020].

Rev. Med. Cine. 2020; 16 (e), 129-136 Ediciones Universidad de Salamanca / @®@ J. Med. Mov., 2020; 16 (e), 129-136 


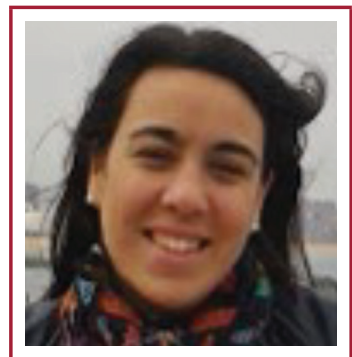

María González García es Diplomada en Enfermería y Fisioterapia. Máster en Enfermería de Urgencias y Cuidados Críticos. Desarrolla su labor asistencial como enfermera de cuidados intensivos. Colaboradora en un proyecto de innovación docente en Grado de Enfermeria de la Universidad de Oviedo. En la actualidad desarrolla su tesis doctoral sobre el estudio de la ciencia, enfermedad y medicamentos en la prensa diaria.

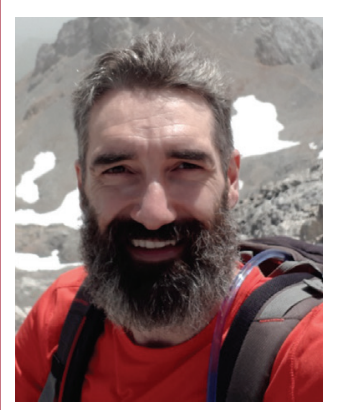

Alberto Lana Pérez. Soy Graduado en Enfermería, Máster en Epidemiologia y Salud Pública y Doctor en Investigación en Medicina. Actualmente ocupo una plaza de Profesor Titular del Área de Medicina Preventiva y Salud Pública de la Universidad de Oviedo, con docencia en los grados de Enfermería, Fisioterapia y Medicina, así como en distintos programas de postgrado. También soy Coordinador del Área de Investigación en Cuidados de Salud del Instituto de Investigación Sanitaria de Asturias e investigador del Grupo de Investigación de Epidemiología Cardiovascular y Nutricional de la Universidad Autónoma de Madrid. He publicado 50 artículos en revistas JCR (Índice H: 13), dirigido 3 tesis doctorales y decenas de trabajos fin de grado y de máster.

Ana Fernández Feito. Diplomada en Enfermería. Licenciada en Antropología Social y Cultural. Doctora (Mención internacional) por la Universidad de

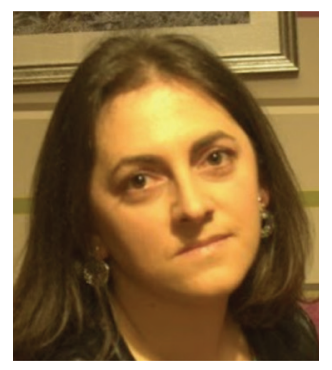
Oviedo. Profesora del Área de Enfermería de la Universidad de Oviedo. Docente en varias asignaturas del Grado en Enfermería, fundamentalmente en Salud Materna y Atención a la Familia y Enfermería del Adulto y Anciano. Responsable del Practicum V (urgencias y cuidados críticos) y VI (hospitalización de adultos/atención primaria de salud).

Responsable del grupo de investigación: Procesos Asistenciales de Enfermería del Instituto de Investigación Sanitaria del Principado de Asturias (ISPA). Líneas de investigación: Valores profesionales en estudiantes y profesionales de enfermería. Docencia y prácticas clínicas en estudiantes de enfermería. Cáncer de mama y detección precoz. Necesidades psicosociales y de información en oncología. 